\title{
Supernormal functional reserve of apical segments in elite soccer players: an ultrasound speckle tracking handgrip stress study Laura Stefani*1, Loira Toncelli1 ${ }^{1}$, Valentina Di Tante ${ }^{1}$, Maria Concetta Roberta Vono ${ }^{1}$, Brunello Cappelli ${ }^{1}$, Gianni Pedrizzetti ${ }^{2}$ and Giorgio Galanti ${ }^{1}$
}

Address: ${ }^{1}$ Sports Medicine Center, Non-invasive cardiac laboratory, University of Florence, Italy and ${ }^{2}$ Dept. Civil and Environmental Engineering, University of Trieste, Italy

Email: Laura Stefani* - laura-stefani@tiscali.it; Loira Toncelli - loiratoncelli@libero.it; Valentina Di Tante - vditante@yahoo.it; Maria Concetta Roberta Vono - mcrvono@libero.it; Brunello Cappelli - bruneca@libero.it; Gianni Pedrizzetti - giannip@dic.units.it;

Giorgio Galanti - g.galanti@dac.unifi.it

* Corresponding author

Published: 16 April 2008

Cardiovascular Ultrasound 2008, 6:14 doi:10.1186/1476-7/20-6-14
Received: 15 January 2008

Accepted: 16 April 2008

This article is available from: http://www.cardiovascularultrasound.com/content/6/I//4

(C) 2008 Stefani et al; licensee BioMed Central Ltd.

This is an Open Access article distributed under the terms of the Creative Commons Attribution License (http://creativecommons.org/licenses/by/2.0), which permits unrestricted use, distribution, and reproduction in any medium, provided the original work is properly cited.

\begin{abstract}
Background: Ultrasound speckle tracking from grey scale images allows the assessment of regional strain derived from $2 \mathrm{D}$ regardless of angle intonation, and it is highly reproducible. The study aimed to evaluate regional left ventricular functional reserve in elite soccer players.

Methods: 50 subjects ( 25 elite athletes and 25 sedentary controls), aged $26 \pm 3.5$, were submitted to an echo exam, at rest and after the Hand Grip (HG) test. Both standard echo parameters and strain were evaluated.

Results: Ejection fraction was similar in athletes and controls both at rest (athletes $58 \pm 2$ vs controls $57 \pm 4 \mathrm{p} \mathrm{ns}$ ) and after HG (athletes $60 \pm 2$ vs controls $58 \pm 3 \mathrm{p} \mathrm{ns}$ ). Basal (septal and anterior) segments showed similar strain values in athletes and controls both at rest (athletes $S \%$ $-19.9 \pm 4.2$; controls $\mathrm{S} \%-18.8 \pm 4.9 \mathrm{p}=\mathrm{ns}$ ) and after HG (athletes $\mathrm{S} \%-20.99 \pm 2.8$; controls $\mathrm{S} \%$ $19.46 \pm 4.4 p=n s$ ). Medium-apical segments showed similar strain values at rest (athletes $S \%-17.31$ \pm 2.3 ; controls $\mathrm{S} \%-20.00 \pm 5.3 \mathrm{p}=\mathrm{ns}$ ), but higher values in athletes after HG (athletes $\mathrm{S} \%-24.47$ \pm 2.8 ; controls $\mathrm{S} \%-20.47 \pm 5.4 \mathrm{p}<0.05$ )

Conclusion: In athletes with physiological myocardial hypertrophy, a brief isometric effort produces enhancement of the strain in medium-apical left ventricular segments, suggesting the presence of a higher regional function reserve which can be elicited with an inotropic challenge and suitable methods of radial function quantification such as $2 \mathrm{D}$-derived strain.
\end{abstract}

\section{Background}

Regional myocardial function is currently evaluated by with 2D echocardiography in the four-chamber view[1] at rest and after pharmacological or physical stress. The evaluation of heart dysfunction, that is often due to ischemia, usually derives from myocardial kinetics modifications[2].

In athletes, where ischemia is an uncommon event, the main information on myocardial performance is related 
to global contractility, normally expressed by left ventricular Ejection Fraction (EF) [3,4].

Traditionally, a 2D echocardiographic exam is used in the four-chamber view for analysing the motion of the segments in the LV near the apex [5]; however this type of assessment can often be problematic due to the poor resolution of the lateral wall [6]. Currently the availability of new non-invasive methods for deformation parameters will make a significant contribution to a more accurate analysis of whole and segmental myocardial performance [7].

The segmental contributions of myocardial systolic function can be evaluated using non-invasive quantification of myocardial strain [8]. Strain is a physically-based measure of myocardial deformation, and it may be a tool for detecting systolic function in physiological hypertrophy $[7,9,10]$. Of the components of strain (longitudinal, circumferential and radial), longitudinal strain is conventionally calculated from the gradient of tissue velocity evaluated with Tissue Doppler Imaging (TDI) [9]. Estimation from TDI is limited to the segments that are wellaligned with the scan line, and it is therefore best applied to basal and medial regions $[10,11]$. Strain can be also estimated with "speckle tracking", a novel non-Dopplerbased method that yields tissue velocity values from grey scale imaging, with high reproducibility of results $[12,13]$ and no angle dependence. While the results of the two methods are consistent when applied to the basal segments of myocardial walls [14], only the latter allows accurate measurement of strain in the left ventricle paraapical-segment $[13,15,16]$. For these reasons, the regional contribution of left ventricular wall segments to overall heart performance has not yet been evaluated, especially when the medium-apical segments of the left ventricle are investigated. Experience led us to hypothesise that the efficiency of an athlete's heart during sporting activity might differ in the different LV segments, and that study of this aspect might allow us to better distinguish the various contributions of the single segments to overall heart performance. The present study thus aims to quantify the Longitudinal Peak Systolic Strain (LPSS) and Longitudinal Velocity (LV) values in a group of athletes at rest and during stress.

\section{Methods}

For reasons of homogeneity, all the subjects studied were men. Twenty-five elite soccer players (ACF Fiorentina, Premier League) aged $26 \pm 3.5$, regularly trained for two hours at approximately $80 \%$ of heart rate five days a week for 10 months a year for 5 years or more, and played one game a week during the regular season. All were matched for age, weight and body surface with 25 controls who perform occasional sporting activity (Table 1 ).
Table I: General data

\begin{tabular}{llcc}
\hline Athletes & & Controls & P \\
\hline Age & $26 \pm 3.5$ & $25 \pm 2.6$ & $\mathrm{~ns}$ \\
Weight Kg & $75 \pm 4.5$ & $67 \pm 3.5$ & $\mathrm{~ns}$ \\
Height cm & $182 \pm 3.2$ & $175 \pm 3.5$ & $\mathrm{~ns}$ \\
Body Surface $\mathrm{m}^{2}$ & $1.96 \pm 0.31$ & $1.86 \pm 0.27$ & $\mathrm{~ns}$ \\
\hline
\end{tabular}

The table shows the values of the general data of the two groups studied

All subjects gave their written consent to the study protocol and all procedures were approved by our Local Ethics Committee.

Subjects in both groups were submitted to anamnesis, including information about drugs and smoking; also to a general examination and cardiological check-up, in order to exclude family history of sudden death, hypertension, diabetes, congenital disease. All the subjects enrolled were evaluated with a standard echocardiograhic exam including systolic and diastolic parameters, as well as LPSS and LV values starting from the four-chamber view.

\section{Study protocol}

Each subject remained at rest for 10 minutes, then was submitted to a trans-thoracic echographic exam at rest and immediately after to Hand-Grip (HG) stress. The subject held the dynamometer in one hand, squeezing the handle at $30 \%$ of their previously-established maximal grip strength for 3 minutes. The choice of employing a short, acute effort such as HG was made in order to create pressure load enhancement in the left ventricle chamber and thus in the deformation of the myocardial wall.

At rest and at the end of the HG test, heart rate (HR), systolic and diastolic blood pressure (SBP;DBP) and standard systolic and diastolic echocardiographic parameters were measured. HG was considered efficient if the mean systolic and diastolic pressure values increased significantly during handgrip as compared to the resting state $(\mathrm{p}<$ 0.05).

\section{Echocardiographic measurements}

Echographic exams were performed at rest and during HG with MyLab 50 (Esaote S.p.A, Florence, Italy) equipped with a $2.5 \mathrm{MHz}$ transducer. In accordance with guidelines [17], the traditional systolic function parameters (Left Ventricle Diastolic diameter (LVDd), Left Ventricle Systolic diameter (LVSd), Inter Ventricular Septum (IVS) thickness, Posterior Wall (PW) thickness, left ventricular Cardiac Mass Index (CMI g/m²), [18] and Ejection Fraction (EF) were calculated. Diastolic function was obtained with pulse wave transmitral Doppler flow, considering E wave velocity, A wave velocity, Iso Volumic Relaxation 
Time (IVRT) and Deceleration Time (DT). Left ventricle images in the four-chamber view were recorded and processed with the software package Esaote-X-Strain included in the echograph.

This software employs the speckle tracking method to automatically evaluate myocardial dynamic properties from two-dimensional B-mode echocardiographic clips $[7,8,10]$ (Fig 1, 2).

The software, based on a grey scale and without any angle dependence, offers the possibility of detecting strain in the basal and medium-apical segments of the left ventricle myocardial walls. Frame rate was kept between 50 and 80 $\mathrm{Hz}$ to optimise the application of speckle tracking for calculating Longitudinal Peak Systolic Strain (LPSS \%), and $\mathrm{LV}(\mathrm{cm} / \mathrm{sec})$ in left ventricle basal and medium-apical segments. We considered the LPSS and the LV values as the means of the values obtained at the basal segments of the interventricular septum and lateral wall and medium-apical sections of the same regions (Fig 1, 2).

\section{Statistical analysis}

Data is presented as means \pm standard deviation. The comparisons between athletes and controls at rest and at peak stress were performed with Student's two-tailed unpaired test $(\mathrm{p}<0.05)$, while the comparison between basal (resting) state and Hand Grip for each group was performed with Student's paired " $t$ " test. Statistical and power analysis were performed using Statview and STATA Stata - Corp 2003 software.
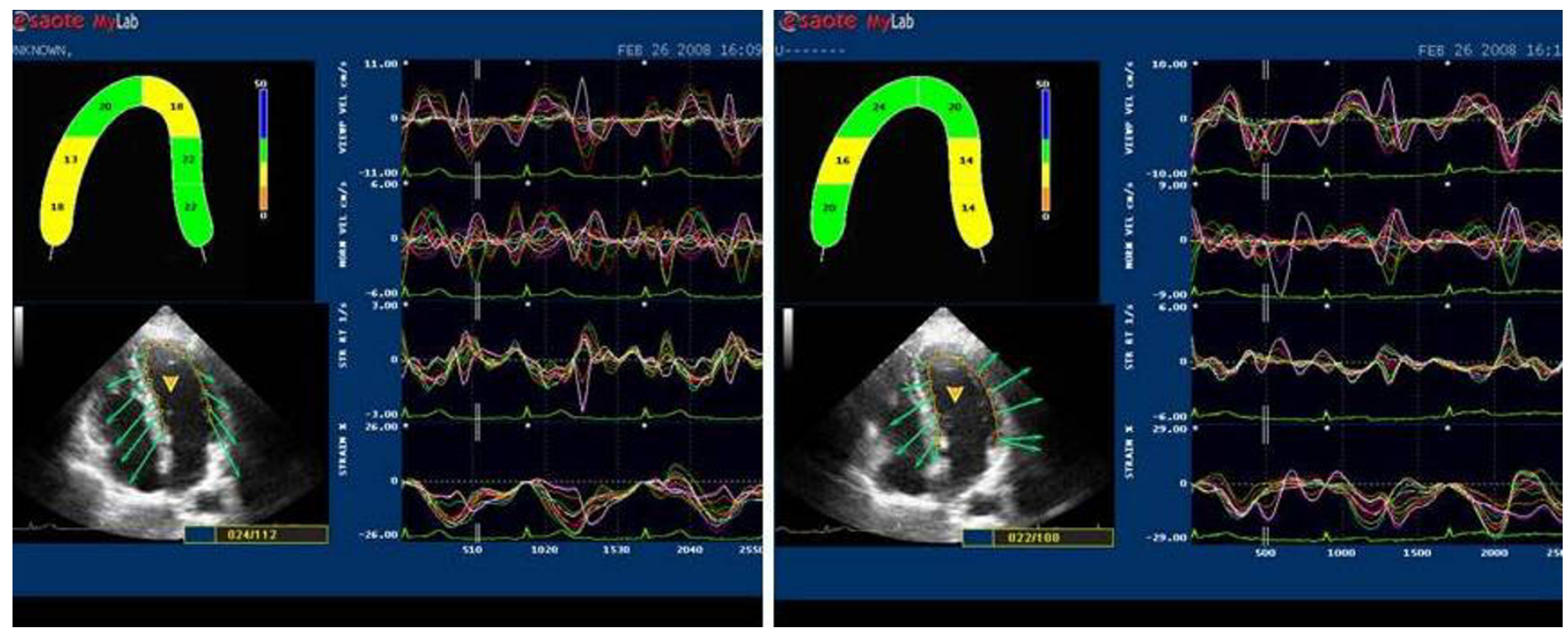

\section{Figure I}

2D LPSS evaluation in athletes' at rest (left side) and after HG (right side). The endocardial border of the 2D left ventricular chamber is automatically followed in time frame by frame. There is no obvious differences in strain in basal and apical segments at rest, but a significant increase after HG. 

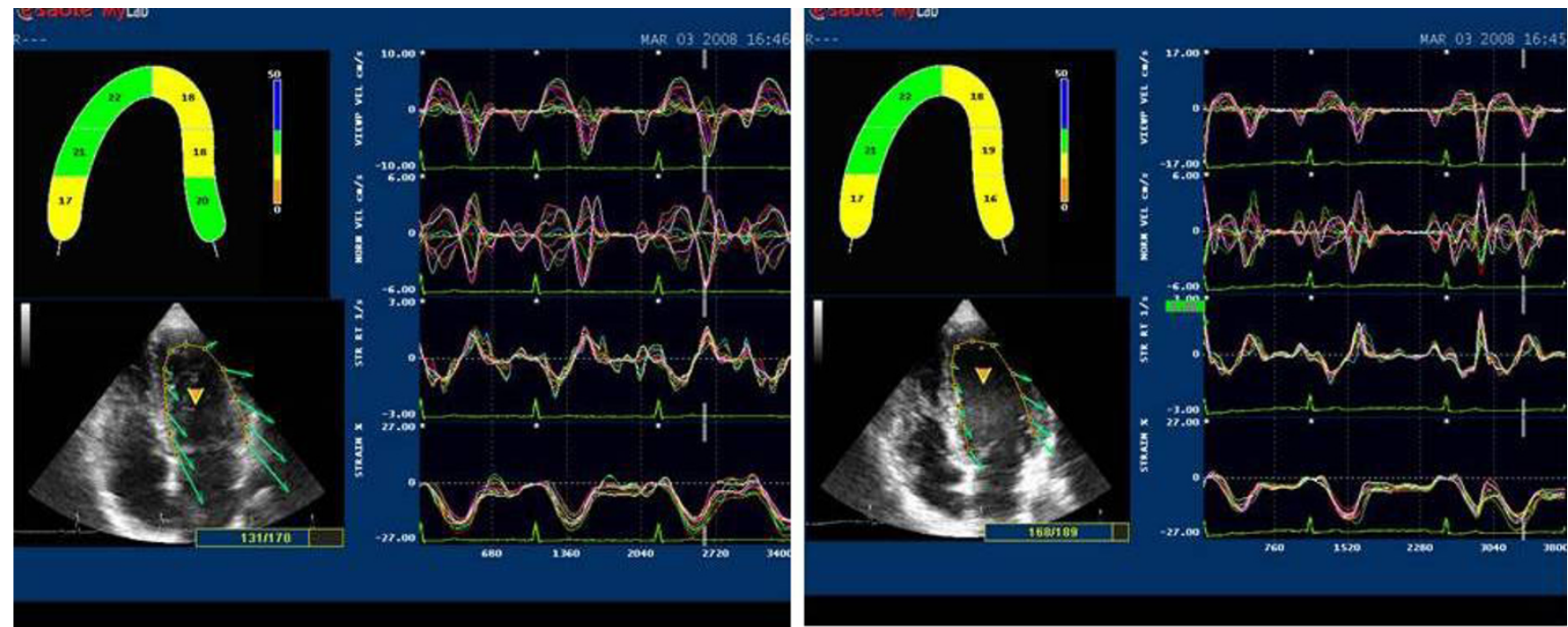

\section{Figure 2}

2D LPSS evaluation at rest (left side) and after HG (right side). Strain values show a slight but not significant increase from basal to apical segments in both situations.

independence $[13,15]$, has enhanced this interest. Commonly, the standard 2D echo exam, has several limitations, like the pitfalls in the evaluation of myocardial performance when it is focussed on the segments close to the heart apex $[5,6]$. On the other hand, 2D-strain evaluation offers high reproducibility of the results with mainly high-quality images.

In athletes, where a major curvature of the walls is present $[3,4]$ as a consequence of physiological hypertrophy, the importance of estimating strain in the left ventricle medium-apical wall segment is linked to the possibility of verifying the involvement of these particular sections in overall myocardial function.

This investigation, aimed to evaluate, using LPSS, the contribution of the regional contractility of left ventricle medium-apical segments to overall heart performance in the athlete with physiological myocardial hypertrophy.

Table 2: Haemodynamic parameters of athletes and controls at rest and after HG

\begin{tabular}{ccccccc}
\hline Athletes & & \multicolumn{5}{c}{ Controls } \\
& rest & HG & P & Rest & HG & P \\
\hline HR & $60 \pm 2$ & $90 \pm 2$ & $<0.05$ & $66 \pm 3$ & $92 \pm 4$ & $<0.05$ \\
DPmmHg & $80 \pm 8$ & $90 \pm 3$ & $<0.05$ & $75 \pm 4$ & $93 \pm 5$ & $<0.05$ \\
SPmmHg & $120 \pm 3$ & $140 \pm 3$ & $<0.05$ & $125 \pm 4$ & $150 \pm 5$ & $<0.05$
\end{tabular}

HR : Heart Rate; DP: Diastolic Pressure; SP : Systolic Pressure; The hemodynamic parameters increase significantly in the two groups after HG.
The results demonstrate the applicability of the new speckle tracking method to the calculation of strain in all sections of the heart, also in the medium -apical segments, with results which come within the normal and validated range [19] A particular effective increase in the LPSS of the left ventricle medium-apical as compared to the basal segments after an HG test is evident in athletes. HG normally produces a pressure overload in the left chamber, and the application of this study protocol enhanced the differences in LPSS values in the athletes' group, showing a gradient between the basal and medium- apical segments which was not recognizable in controls. No significant modification of EF is evident in these parameters in either groups studied after HG.

\section{Conclusion}

These results point to the importance of considering myocardial deformation parameters in LV segments adjacent to the apex, particularly in athletes where regular training produces increased myocardial contractility. We must remember that the actual performance of the apical segments is not correctly assessable with standard 2D; also the current application of the method of strain estimation by TDI is normally limited to basal and medial wall regions due to its angle dependence.

In this study, the measurements of myocardial fibre velocity values in the same segments where strain was determined were made with the speckle tracking method, thus overcoming the limitations of aligning the beam with the scan line. 
Table 3: Echocardiographic parameters of athletes and controls

\begin{tabular}{|c|c|c|c|c|c|c|}
\hline & \multicolumn{2}{|c|}{ Athletes } & \multirow[t]{2}{*}{$P$} & \multicolumn{2}{|c|}{ Controls } & \multirow[t]{2}{*}{$p$} \\
\hline & rest & HG & & rest & HG & \\
\hline IVSmm & $9.27 \pm 0.93$ & $9.7 \pm 1$ & ns & $8.55 \pm 1.2$ & $9.1 \pm 1$ & ns \\
\hline PWmm & $9.17 \pm 0.89$ & $10.1 \pm 0.8$ & ns & $8.64 \pm 1.3$ & $9.8 \pm 0.9$ & ns \\
\hline LVEDdmm & $50.37 \pm 4.11$ & $49.2 \pm 3$ & ns & $49.63 \pm 4.43$ & $48.5 \pm 3$ & ns \\
\hline LVESdmm & $32.25 \pm 3.53$ & $31.4 \pm 3$ & ns & $28.45 \pm 4.18$ & $29.5 \pm 3$ & ns \\
\hline CMI gr/m2 & $101.54 \pm 2$ & $100.21 \pm 1$ & ns & $92.100 \pm 1$ & $91.200 \pm 1$ & ns \\
\hline $\mathrm{LA} \mathrm{mm}$ & $35.12 \pm 1.2$ & $34 \pm 2.3$ & ns & $36.10 \pm 1.3$ & $36.80 \pm 1.0$ & ns \\
\hline IVRT ms & $81.0 \pm 7.4$ & $83.6 \pm 6.4$ & ns & $85.0 \pm 4.0$ & $86.6 \pm 3.0$ & ns \\
\hline DT $m s$ & $190.5 \pm 13.4$ & $178.5 \pm 12.2$ & ns & $170.4 \pm 13.5$ & $180.4 \pm 12.0$ & ns \\
\hline$E / A$ & $1.4 \pm 0.3$ & $1.33 \pm 0.9$ & ns & $1.3 \pm 0.3$ & $1.2 \pm 0.7$ & ns \\
\hline $\mathrm{EF} \%$ & $58 \pm 2$ & $60 \pm 2$ & ns & $57 \pm 4$ & $58 \pm 3$ & ns \\
\hline
\end{tabular}

IVS interventricular septum; PW posterior wall; LVEDd Left Ventricle End Diastolic diameter; LVESd Left Ventricle End Systolic diameter; CMI Cardiac Mass Index; LA :Left Atrium; IVRT Iso Volumetric Relaxation Time; DT Deceleration Time; E/A E-wave A-wave peak velocity ratio; EF Ejection Fraction; HR Heart Rate ; SP Systolic Pressure; DP Diastolic Pressure.

The table shows the values of the main echocardiographic systolic and diastolic parameters at rest and after HG in athletes and controls. No statistical differences are evident.

Our results show a physiological gradient with a decrease in values from basal to apical segments in the two groups, confirming the literature data

In conclusion, the significant increase in LV longitudinal strain in athletes suggests the importance of evaluating deformation parameters in order to more thoroughly assess those morphological and functional modifications in the heart which cannot be discovered by traditional echo measurements.

In agreement with the normal values recently reported in the literature [19], these results, also suggest the possibility of a routine application of these parameters in order to evaluate the athlete's heart and also to define myocardial performance in several other pathologies (involving athletes and patients alike) where heart contraction is of differing degrees according to the different wall segments.

In athletes, this evaluation might be extended in future to the investigation of the strain of the two ventricles contracting in real time, in order to determine a the different behaviour of value of these two chambers that normally work at different load pressures.

\section{Limitation of the study}

This investigation was performed in athletes with highquality myocardial echo-images and no further information is available about the possibility of obtaining the same results with high reproducibility in other conditions where image resolution is poor. The endocardial border needs to be optimal, with a clear delineation of the myocardial tissue and extracardiac structures.

We must also remember that myocardial contractility is a complex mechanism, recently addressed with new sophisticated software evaluating all components of wall deformation, like radial strain and torsion. In this study the evaluation of the deformation of segments around the myocardial apex, which is normally difficult from the standard 2D echocardiographic four-chamber view[1] can however be performed with a new non Doppler-derived method, speckle tracking. In addition, considering the difficulty of approaching this region using standard TDI, the

Table 4: Longitudinal Peak Systolic Strain (LPSS) and Longitudinal Velocity (LV) in athletes

\begin{tabular}{|c|c|c|c|c|c|c|}
\hline \multicolumn{7}{|l|}{ Athletes } \\
\hline & \multicolumn{2}{|c|}{ Medium-Apical segments } & \multirow[t]{2}{*}{$p$} & \multicolumn{2}{|c|}{ Basal segments } & \multirow[t]{2}{*}{$p$} \\
\hline & rest & HG & & rest & HG & \\
\hline $\mathrm{LV} \mathrm{cm} / \mathrm{s}$ & $-0.93 \pm 0.5$ & $-1.40 \pm 0.6$ & $<0.05$ & $-5.00 \pm 1.6$ & $-5.01 \pm 1.3$ & ns \\
\hline LPSS $\%$ & $-17.31 \pm 2.3$ & $-24.47 \pm 2.8$ & $<0.05$ & $-19.9 \pm 4.2$ & $-20.99 \pm 2.8$ & ns \\
\hline
\end{tabular}

$\mathrm{V}(\mathrm{cm} / \mathrm{s})$ Velocity; LPSS\% Longitudinal Peak Systolic Strain. In athletes myocardial strain and velocity values increase significantly in medium-apical segments during $\mathrm{Hg}$ 
Table 5: Longitudinal Peak Systolic Strain (LPSS) and Longitudinal Velocity (LV) values in controls

\begin{tabular}{|c|c|c|c|c|c|c|}
\hline \multicolumn{7}{|l|}{ Controls } \\
\hline & \multicolumn{2}{|c|}{ Medium-Apical segments } & \multirow[t]{2}{*}{$p$} & \multicolumn{2}{|c|}{ Basal segments } & \multirow[t]{2}{*}{$p$} \\
\hline & Rest & HG & & rest & HG & \\
\hline $\mathrm{Vcm} / \mathrm{s}$ & $-1.81 \pm 0.2$ & $-1.43 \pm 1.2$ & ns & $-5.73 \pm 0.9$ & $-4.20 \pm 0.9$ & ns \\
\hline LPSS \% & $-20.00 \pm 5.3$ & $-20.47 \pm 5.4$ & ns & $-18.8 \pm 4.9$ & $-19.46 \pm 4.4$ & ns \\
\hline
\end{tabular}

$\mathrm{V}(\mathrm{cm} / \mathrm{s})$ Velocity ; LPSS\% Longitudinal Peak Systolic Strain. In controls, the values do not change.

investigation also included the non-Doppler velocity profile, which showed a base medium apex velocity gradient in each wall.

Although the contribution of the contractility of left ventricle medium apical segments was found to be higher than in the basal ones in soccer players submitted to an HG test, no more information about the impact of a different kind of effort, different sport or different training level is yet available. For this reason, this study must be considered as a partial investigation, which however signals the usefulness of further studies to complete performance evaluation in the athlete's heart

\section{Competing interests}

The author(s) declare that they have no competing interests.

\section{Authors' contributions}

The initial concept of the study was of GG and LS, who designed the study. LS, GP, LT performed all the measurements and statistical analyses. LS wrote the manuscript and all the authors contributed to, read, and approved the final version.

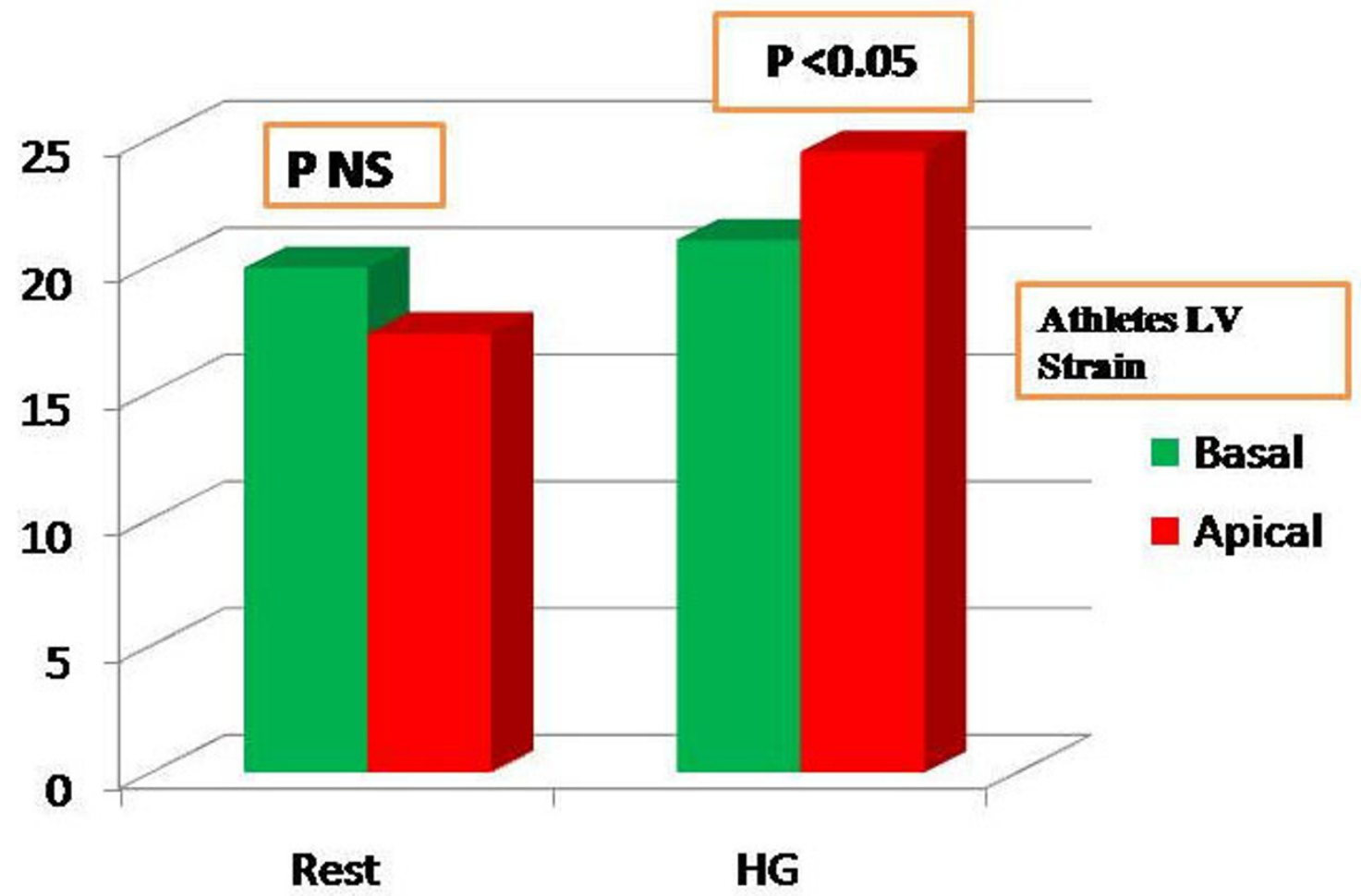

Figure 3

LPSS(\%) values of basal (green) and medium-apical (red) left ventricle segments in athletes at rest and after HG. A significant increase is evident in medium-apical segments after HG. The strain values, currently negative, are conventionally expressed in positive in this graph. 


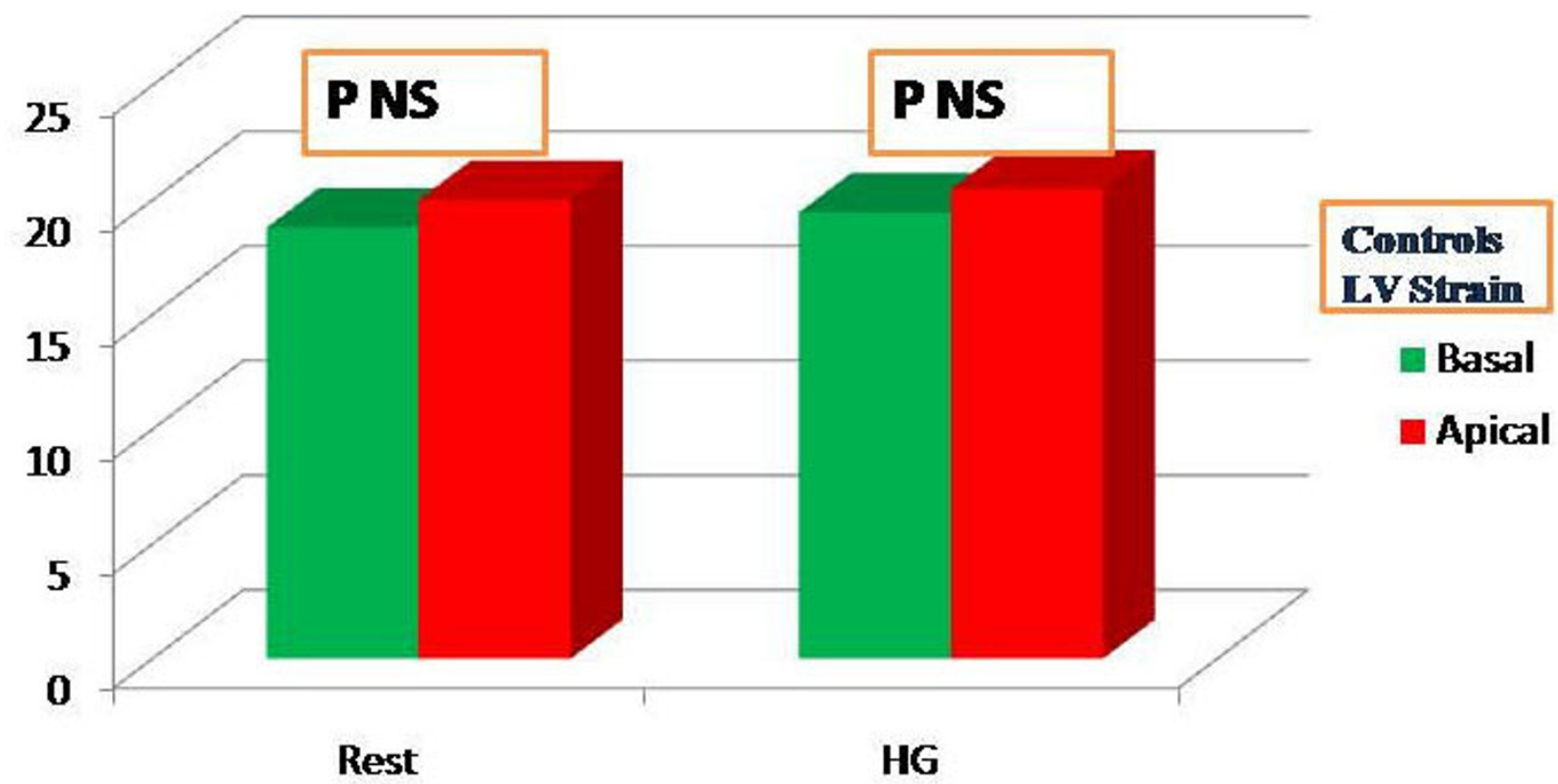

Figure 4

LPSS(\%) values of basal (green) and medium-apical (red) left ventricle segments in controls at rest and after HG. No significant increase is evident at rest and after HG in the segments analyzed. The strain values, currently negative, are conventionally expressed in positive in this graph.

\section{Acknowledgements}

The abstract was accepted as an oral presentation at Euroecho 10 (Prague, Dec 6-9 2006) and at the conference of the Italian Society of Cardiology (Rome, Dec 16-19 2006)

We wish to thank Prof Vieri Boddi for his statistical support and Susan Charlton for her revision of the English manuscript.

\section{References}

I. Nosir YFM, Vletter WB, Boersma E, Frowjin R, Ten Cate Fj: The apical long-axis rather than the two chamber should be in combination with the four chamber view to accurate assessment of left ventricular volumes and function. European Heart Journal 2000, 18: I I75-II85.

2. Feigenbaum H, Armostrong WF, Ryan T: Feigenbaum's Echocardiography Sixth edition. Lippincott Williams \& Wilkins; 2005.

3. Shephard RJ: The athlete's heart: is big beautiful? Br J Sports Med 1996, 30:5-10.

4. Pluim BM, Zwinderman AH, Laarse A, Wall E: The athlete's heart: a Meta Analysis of cardiac structure and function. Circ 1999 , I 01 (3):336-344.

5. Picano E: Stress Echocardiography 4th edition. Springer-Verlag Berlin Heidelberg; 2003.

6. Hirata K, Watanabe H, Beppu S, Muro T, Teragaki M, Yoshiyama M, Takeuchi K, Yoshikawa J: Pitfalls of echocardiographic measurement in tissue harmonic imaging: in vitro and in vivo study. J Am Soc Echocardiogr 2002, I 5:1038-44.

7. Serri K, Reant P, Lafitte M, Berhouette M, Le Bouffos V, Roudaut R, Lafitte S: Global and regional myocardial function quantification by two dimensional strain. JACC 2006, 47(6): I I75-II8I.

8. Stig U, Thor E, Hans T, Bjorn A, Otto AS: Myocardial strain by Doppler echocardiography. Validation of a new method to quantify regional myocardial function. Circ 2000, 102:1158-1164.
9. Greenberg N, Firstenberg MS, Castro PL, Main M, Travaglini A, Odabashian JA, Drinks JK, Rodriguez LI, Thomas JD, Garcia MJ: Dopplerderived Myocardial Systolic Strain rate. is a strong index of left ventricular contractility. Circ 2002, 105:99-105.

10. Souterland GR, Di Salvo G, Claus P, D'Hoogle J, Bijnens B: Strain and strain rate imaging a new clinical approach to quantifying regional myocardial function. J Am Soc Echocardiogr 2004, 17:788-802.

II. Urheim S, Edvardsen T, Torp H, Angelsen B, Smiseth OA: Myocardial strain by Doppler echocardiography. Validation of a new method to quantify regional myocardial function. Circ 2000 , 102(10): I I58-II64.

12. Kirkhon J, Bajarmen S, Olstad B, Kristofersen K, Torp HA: A new technique for improved spatial resolution in high-frame rate color Doppler imaging. IEEE 2003, I: 1947-50.

13. Stoylen A: Strain rate imaging of the left ventricle. Doctoral Thesis, NTNU, Stockholm 200I [http://folk.ntnu.no/stoylen/strainrate/].

14. Stefani L, Toncelli L, Gianassi M, Manetti P, Di Tante V, Vono MCR, Cappelli B, Pedrizzetti G, Galanti G: Two-dimensional tracking and TDI are consistent methods for evaluating myocardial longitudinal peak strain in left and right ventricle basal segments in athletes. Cardiovascular Ultrasound 2007, 7(5):7.

15. Stefani L, Di Tante V, Mercuri R, Ingarozza A, De Luca A, Toncelli L, Vono MCR, Mnanetti P, Caselli L, Gianassi M, Pedrizzetti G, Galanti G: Real time evaluation of longitudinal peak systolic strain of left and right ventricle after an isometric test. Behaviour in athletes. Circ 2007, I 16:11-449.

16. Richand V: An ultrasound speckle tracking(two dimensional strain) analysis of myocardial deformation in professional soccer players compared with healthy subjects and hypertrophic cardiomiopathy. Am / Cardiol 2007, I 00: | 28-32.

17. Cheitilin MD, Armstrong WF, Aurigemma GR: 2003 Guideline update for the clinical application of echocardiography : A report of the American College of Cardiology, American Heart Association task force on practice guidelines. J Am Soc Echocardiography 2003, 16:1091-110. 
18. Devereux RB: Echocardiographic assessment of left ventricular hypertrophy comparison with necroscopy findings. Am Heart J 1986, 57:450-8.

19. Kowalski M, Kukulski T, Jamal F, D'Hoogge J, Widemann F, Rademakers F, Bijnens B, Hatle L, Sutherland GR: Can natural strain and strain rate quantify regional myocardial deformation? A study in healthy subjects. Ultrasound in Med \& Biol 200I, 27:1087-97.

Publish with Biomed Central and every scientist can read your work free of charge

"BioMed Central will be the most significant development for disseminating the results of biomedical research in our lifetime. " Sir Paul Nurse, Cancer Research UK

Your research papers will be:

- available free of charge to the entire biomedical community

- peer reviewed and published immediately upon acceptance

- cited in PubMed and archived on PubMed Central

- yours - you keep the copyright

Submit your manuscript here:

http://www.biomedcentral.com/info/publishing_adv.asp 